\title{
New Method for Determination of Diffusion Coefficients in Turbulent Plasmas
}

\author{
Alex Ivascenko* \\ Centre for Space Research, North-West University, Potchefstroom, South Africa \\ E-mail: $24790052 @$ nwu.ac.za \\ Felix Spanier \\ Centre for Space Research, North-West University, Potchefstroom, South Africa \\ E-mail: felixafspanier.de

\section{Urs Ganse} \\ Department of Physics, University of Helsinki, Finland \\ E-mail: urs.ganse@helsinki.fi
}

\begin{abstract}
The transport of charged particles in turbulent magnetic fields is a topic of great interest in astrophysics, since our ability to successfully use cosmic rays as astronomic messengers depends on our understanding of the transport processes. One of the primary effects is the scattering of particles on magnetic irregularities leading in the first instance to a change in the pitch angle $\mu$ of the particle. Further transport parameters e.g. diffusion coefficients or the mean free path can then be inferred from $\Delta \mu$. We introduce new numerical methods to evaluate the pitch angle diffusion coefficient $D_{\mu \mu}$ and the perpendicular diffusion coefficient $D_{\perp}$ that work well in both weak and strong turbulence scenarios and compare with analytic results from Quasi Linear Theory.

We present results obtained by applying these methods to test-particle data from simulation of heliospheric conditions with our spectral MHD code Gismo. The results are then compared to test-particle simulations of the novel Perlin-noise-type pseudo-turbulence to show its viability for large-scale particle transport simulations.
\end{abstract}

The 34th International Cosmic Ray Conference,

30 July- 6 August, 2015

The Hague, The Netherlands

\footnotetext{
*Speaker.
} 


\section{Introduction}

The motion of charged particles in electromagnetic fields beyond the trivial case of gyration/drift in homogeneous static fields is governed by resonant wave-particle-interactions that result in particles being scattered on electromagnetic irregularities. In guiding center coordinates this process can be described reasonably well as a change of the particle's pitch angle $\mu$ and, assuming an underlying diffusive nature of the process, characterized with the pitch angle diffusion coefficient

$$
D_{\mu \mu}=\lim _{t \rightarrow \infty} \frac{(\Delta \mu)^{2}}{2 \Delta t} .
$$

This coefficient can be derived analytically by applying Quasi Linear Theory (QLT), that was first suggested by [1] and is primarily based on the assumption of unperturbed particle orbits, which is only valid for weak turbulence $(\delta B / B \ll 1)$. In this paper we present new methods to determine the coefficient in strong and weak turbulence scenarios and apply them to numerical simulation data, underlining its importance for observable quantities and the mean free path of a charged particle in a plasma.

\section{Particle transport}

The time development of a particle distribution or phase space density $f_{T}$ of particle species $T$ under the influence of the Lorentz force is described by the relativistic Vlasov equation

$$
\frac{\partial f_{T}}{\partial t}+\frac{\vec{p}}{m \gamma} \cdot \frac{\partial f_{T}}{\partial \vec{x}}+\frac{q}{c}(c \vec{E}(\vec{x}, t)+\vec{v} \times \vec{B}(\vec{x}, t)) \cdot \frac{\partial f_{T}}{\partial \vec{p}}=S_{T}(\vec{x}, \vec{p}, t)
$$

with a source term $S_{T}$ on the RHS.

Using the fact that a charged particle in a magnetic field performs a gyration with the frequency $\Omega=\frac{Z e B}{\gamma m c}$ the equation can be transformed into guiding centre coordinates $(X, Y, Z, p, \mu, \phi)$ with $\mu$ the cosine of the pitch-angle between the particle momentum vector and the magnetic field and $\phi$ in the plane perpendicular to the magnetic field. Taking the ensemble average under QLT's assumptions of unperturbed particle orbits yields the Fokker-Planck equation for $F_{T}=\left\langle f_{T}\right\rangle$ :

$$
\frac{\partial F_{T}}{\partial t}+v \mu \frac{\partial F_{T}}{\partial Z}-\Omega \frac{\partial F_{T}}{\partial \phi}=S_{T}\left(X_{\sigma}, t\right)+\frac{1}{p^{2}} \frac{\partial}{\partial X_{\sigma}}(p^{2} \frac{\partial F_{T}}{\partial \hat{X}_{\sigma}} \underbrace{\int_{0}^{t} \mathrm{~d} s\left\langle g_{X_{\sigma}} g_{\hat{X}_{\sigma}}\left(\hat{X}_{\sigma}, s\right)\right\rangle}_{D_{X_{\sigma} X_{\sigma}}}) .
$$

The time integral over the generalized forces $g_{X_{\sigma}}=\dot{X}_{\sigma}$ in the last term on the RHS results in the Fokker-Planck coefficients $D_{X_{\sigma} \hat{X}_{\sigma}}$ (for a complete derivation see [2]).

Due to the diffusive nature of the term the Fokker-Planck coefficients take the role of diffusion coefficients and describe the scattering of particles in 6-dimensional phase space. Our primary interest lies with the pitch angle diffusion coefficient $D_{\mu \mu}$ that describes the pitch angle scattering of the particle with the velocity $v_{\|}$and gyro-frequency $\Omega$ in resonant interactions with a wave with frequency $\omega$ and wavenumber $k_{\|}$that fulfill the condition

$$
k_{\|} v_{\|}-\omega+n \Omega=0, \quad n \in \mathbb{Z} .
$$


The order of the interaction $n$ corresponds to contributions from different wave components. The Cherenkov resonance with $n=0$ is generated by pseudo Alfvén waves through the mirror force induced by magnetic compressions. The parallel component of shear Alfvén waves will fulfill Eq. 2.3 for $n= \pm 1$. Interactions with $|n|>1$ can only be generated by oblique Alfvén waves. The perpendicular components of the wave would then modify the scattering process by non-vanishing Bessel functions.

\section{Methods}

The presented analysis is based on numerical results of spectral incompressible MagnetoHydro-Dynamic (MHD) simulations with Gismo [3, 4] and Particle-in-Cell (PiC) simulations with Acronym [5]. Using a test particle approach yields particle trajectories $\vec{x}(t)$ from which the phase space density $f(\vec{x}, \vec{p})$ and time-dependent pitch angle $\mu(t)$ can be determined.

In the following sections we first recap the common QLT approach to derive the diffusion coefficient $D_{\mu \mu}$ for comparison with our new methods that are presented afterwards.

\subsection{Running diffusion coefficient}

A simple approach for calculating the pitch angle scattering coefficient is the definition

$$
D_{\mu \mu}=\lim _{t \rightarrow \infty} \frac{(\Delta \mu)^{2}}{2 \Delta t} \stackrel{t \gg t_{0}}{\approx} \frac{(\Delta \mu)^{2}}{2 \Delta t},
$$

where $\Delta t=t-t_{0}$ is assumed to be large, i.e. the time evolution $t$ has to be sufficient to develop resonant interactions. This approach is motivated by a description of diffusion, where a particle changes its pitch angle by scattering in a randomized process. If the scattering is in resonance with a wave mode, $\Delta \mu$ is significantly increased. This method predicts a $\delta$-function shape in the limit of infinite time development. However, in finite intervals of $\Delta t$ the resonances are always broadened. Another problem is the dependency on the strength of the scattering process. In the case of high $\delta B / B_{0}$ ratios and thus high scattering frequencies, the pitch angle is not connected to its initial state anymore and the scattering coefficient becomes unstructured [6].

\subsection{MIIa Diffusion equation fitting method}

A completely different approach is the calculation via the diffusion equation. The basic concept is the assumption that the pitch angle diffusion is the predominant process. Eq. 2.2 then becomes

$$
\frac{\partial f_{T}}{\partial t}-\frac{\partial}{\partial \mu} D_{\mu \mu} \frac{\partial f_{T}}{\partial \mu}=0
$$

This allows us to calculate the diffusion coefficient from the static particle distribution in $\mu$-space at two distinct timestamps by solving the diffusion equation

$$
\frac{\partial f_{T}(\mu, t)}{\partial t}=\left(\frac{\mathrm{d}}{\mathrm{d} \mu} D_{\mu \mu}(\mu)\right) \cdot \frac{\partial f_{T}(\mu, t)}{\partial \mu}+D_{\mu \mu}(\mu) \cdot \frac{\partial^{2} f_{T}(\mu, t)}{\partial \mu^{2}}
$$


numerically for $D_{\mu \mu}(\mu)$.

Since the simulations provide us with discrete distributions, the derivatives are discretized accordingly in the usual way, yielding an equation for every $\mu^{n}=-1+n \cdot \Delta \mu$ (with $D_{\mu \mu}^{n}=D_{\mu \mu}\left(\mu^{n}\right)$ ):

$$
\partial_{t} f=\frac{D_{\mu \mu}^{n+1}-D_{\mu \mu}^{n-1}}{2 \cdot \Delta \mu} \partial_{\mu} f+D_{\mu \mu}^{n} \partial_{\mu \mu} f
$$

This corresponds to a matrix equation with a tridiagonal matrix which can be solved with conventional algorithms:

$$
\left(\begin{array}{cccc}
\partial_{\mu \mu} f^{0} & \frac{\partial_{\mu} f^{0}}{2 \Delta \mu} & 0 & 0 \\
-\frac{\partial_{\mu} f^{1}}{2 \Delta \mu} & \partial_{\mu \mu} f^{1} & \ddots & 0 \\
0 & \ddots & \ddots & \frac{\partial_{\mu} f^{n-1}}{2 \Delta \mu} \\
0 & 0 & -\frac{\partial_{\mu} f^{n}}{2 \Delta \mu} & \partial_{\mu \mu} f^{n}
\end{array}\right) \cdot\left(\begin{array}{c}
D_{\mu \mu}^{0} \\
D_{\mu \mu}^{1} \\
\vdots \\
D_{\mu \mu}^{n}
\end{array}\right)=\left(\begin{array}{c}
\partial_{t} f^{0} \\
\partial_{t} f^{1} \\
\vdots \\
\partial_{t} f^{n}
\end{array}\right)
$$

A problem of this method is the imperfect sampling of the phase space with the test particle approach, which results in a rather noisy distribution function and even noisier derivatives. This can be handled by averaging over several simulation runs, applying smoothing algorithms or fitting the data with analytical functions.

While the ensemble averaging is the correct way to increase the signal-to-noise ratio, it would mean a huge computational effort to obtain more simulation data and can't be applied at all to real measurements. We found that fitting the distribution function with a generic polynomial that reproduces the main features and sampling the analytical derivatives yields the best results. Alternatively, smoothing the data with a Gaussian kernel with appropriately chosen width also leads to satisfactory results.

\subsection{MIIb Diffusion equation integration method}

Another way to deal with the noisy derivatives is to integrate the diffusion equation numerically over $\mu$

$$
\int_{-1}^{\mu} \frac{\partial f_{T}(\mu, t)}{\partial t} \mathrm{~d} \mu=D_{\mu \mu}(\mu) \frac{\partial f_{T}(\mu, t)}{\partial \mu}=-j_{\mu}(\mu)
$$

thus gaining the effective pitch angle current $j_{\mu}$ that yields the diffusion coefficient when divided by $\partial_{\mu} f_{T}$. The advantage of this method is that the time derivative of $f_{T}$ is smoothed by the integration and we only need the first derivative in $\mu$, which can also be approximated by a polynomial if necessary.

\section{Simulation setup}

The MHD simulation setup was chosen to resemble the conditions in the solar corona at a distance of three solar radii. The anisotropic turbulence is driven continuously by injecting energy at low wave numbers. The magnetic background field $B_{0}=0.174 \mathrm{G}$ and particle density of $10^{5} \mathrm{~cm}^{-3}$ result in an Alfvén speed of $v_{A}=1.2 \cdot 10^{8} \mathrm{~cm} \mathrm{~s}^{-1}$ [7]. The outer length scale of the simulated system is $L_{\text {scale }}=3.4 \cdot 10^{8} \mathrm{~cm}$. 
The turbulence driving is performed by allocating an amplitude with a phase to the fields within the Fourier space. The amplitude follows a power-law of $|\vec{k}|^{-2.5}$ and is initialized using a normal distribution. The phase was randomly chosen between zero and $2 \pi$. These settings are divergencefree and Hermitian symmetric. After this initialization the values were scaled to the desired scenario, which in our case is a $\delta B / B_{0}$ ratio of roughly $10^{-2}$. Note that both species, pseudo- and shear Alfvén waves, are excited by this type of turbulence driving. In this inertial range energy is injected every $0.03 \mathrm{~s}$ (10 MHD-steps), which leads to a saturated turbulence - an equilibrium between dissipation and injection.

At this stage $10^{6}$ proton-like test particles were injected, initialized with random positions $\vec{x}$ and $\phi$. For the initial distribution in $\mu$ a half-parabola was chosen, since the methods MII require a non-vanishing $\mu$-derivative. The proton speed was set to a value of $1.21 \cdot 10^{10} \mathrm{~cm} \cdot \mathrm{s}^{-1}$, which was chosen to fulfill the resonance condition (Eq. 2.3).

\section{Results}

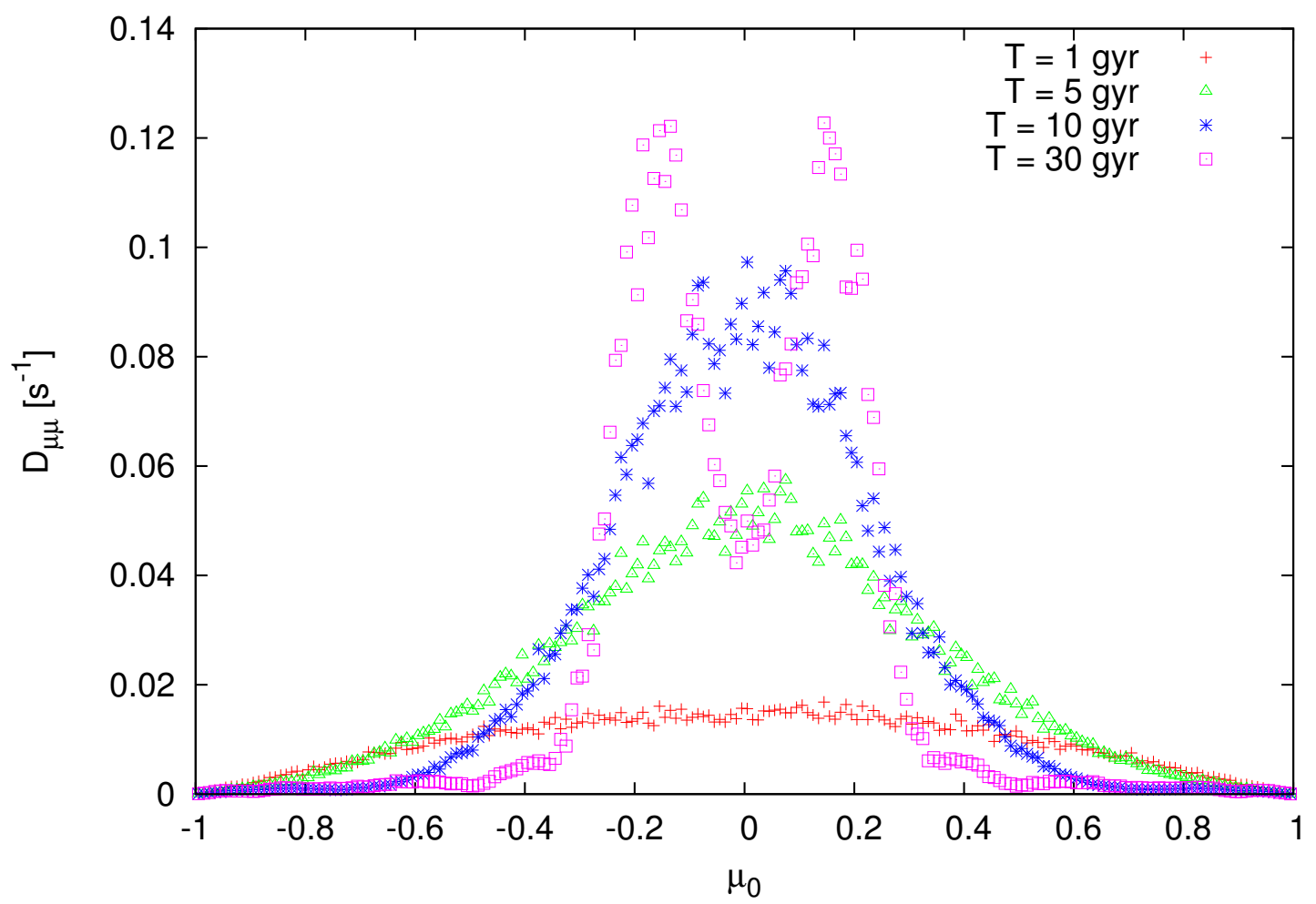

Figure 1: Time evolution of the pitch angle scattering coefficient $D_{\mu \mu}$ calculated by MI. A clear resonant structure develops between 10 and 30 gyration periods. The maxima can be connected to the Cherenkov resonance.

First we present the pitch angle diffusion coefficient $D_{\mu \mu}$ obtained with the classical running coefficient method MI. In Fig. 1 the development of a resonant structure is clearly visible as the 
simulation progresses from 1 to 30 gyration periods. It's important to note that this development is not a physical property of the turbulence, but merely a stochastic effect of the increasing portion of the homogeneously distributed particles interacting resonantly with the wave modes running through the simulation box. This should not be confused with the development of the turbulence itself which is completed before test particles are injected into the simulation.

Another thing to note here is the apparent splitting of the maxima at 30 gyration periods. This is caused by the tilt of the sharp resonance peak in $\Delta \mu$ when plotted over the initial $\mu_{0}$. Calculating $(\Delta \mu)^{2}$ folds the negative half-peak up resulting in the apparent double-peak structure. The correct choice of the starting pitch angle $\mu_{0}$ has been discussed in [8] and while this can improve the appearance of the plot, it does not change its fundamental meaning.

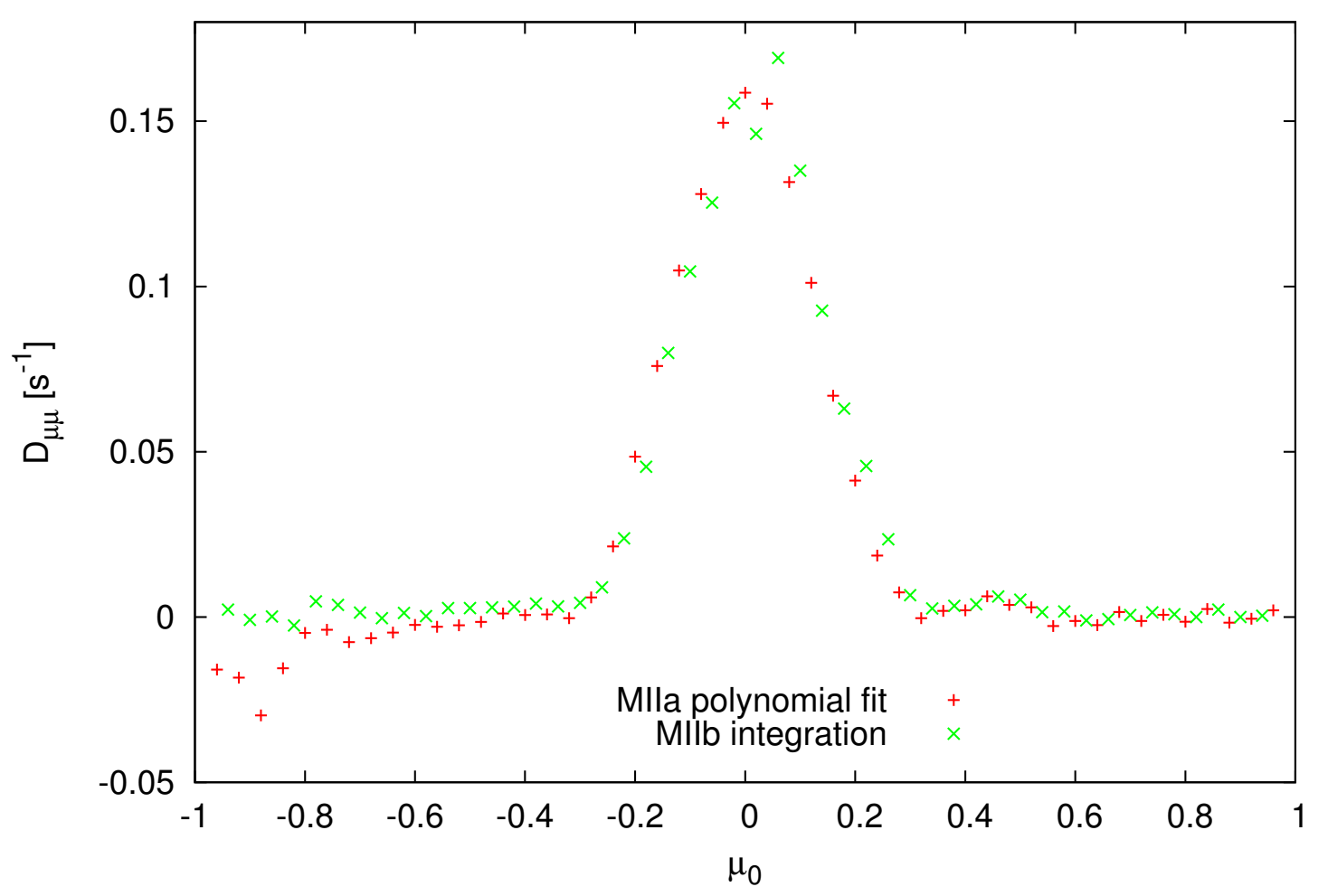

Figure 2: Comparison of MIIa and MIIb for the background turbulence simulation clearly showing the Cherenkov resonance. The shown curves correspond to a simulation time of 10 gyration periods.

In Fig. 2 we present $D_{\mu \mu}$ obtained with our new methods MIIa and MIIb from the same simulation setup. The results of the direct integration MIIb match the solution of the matrix equation MIIa almost perfectly and the width and height of the Cherenkov resonance peak compare very well to MI in Fig. 1. Since these methods work with the pitch angle distribution $f_{T}(\mu)$ rather than with the pitch angle change $\Delta \mu$, the maximum does not split, thus the results from MIIa and MIIb can be used directly without any interpretive help.

A problem of these methods is the requirement towards the distribution function $f_{T}(\mu)$, namely the non-vanishing $\mu$-derivative. While this is relatively easy to fulfill in test particle simulations with a 
half-parabola distribution, it leads to further problems with particle statistics, the results of which can be seen near $\mu_{0}=-1$ in Fig. 2 where the particle number is at its minimum. This problem can be mitigated somewhat by adding a constant background to the particle distribution and by averaging over runs with mirrored distributions.

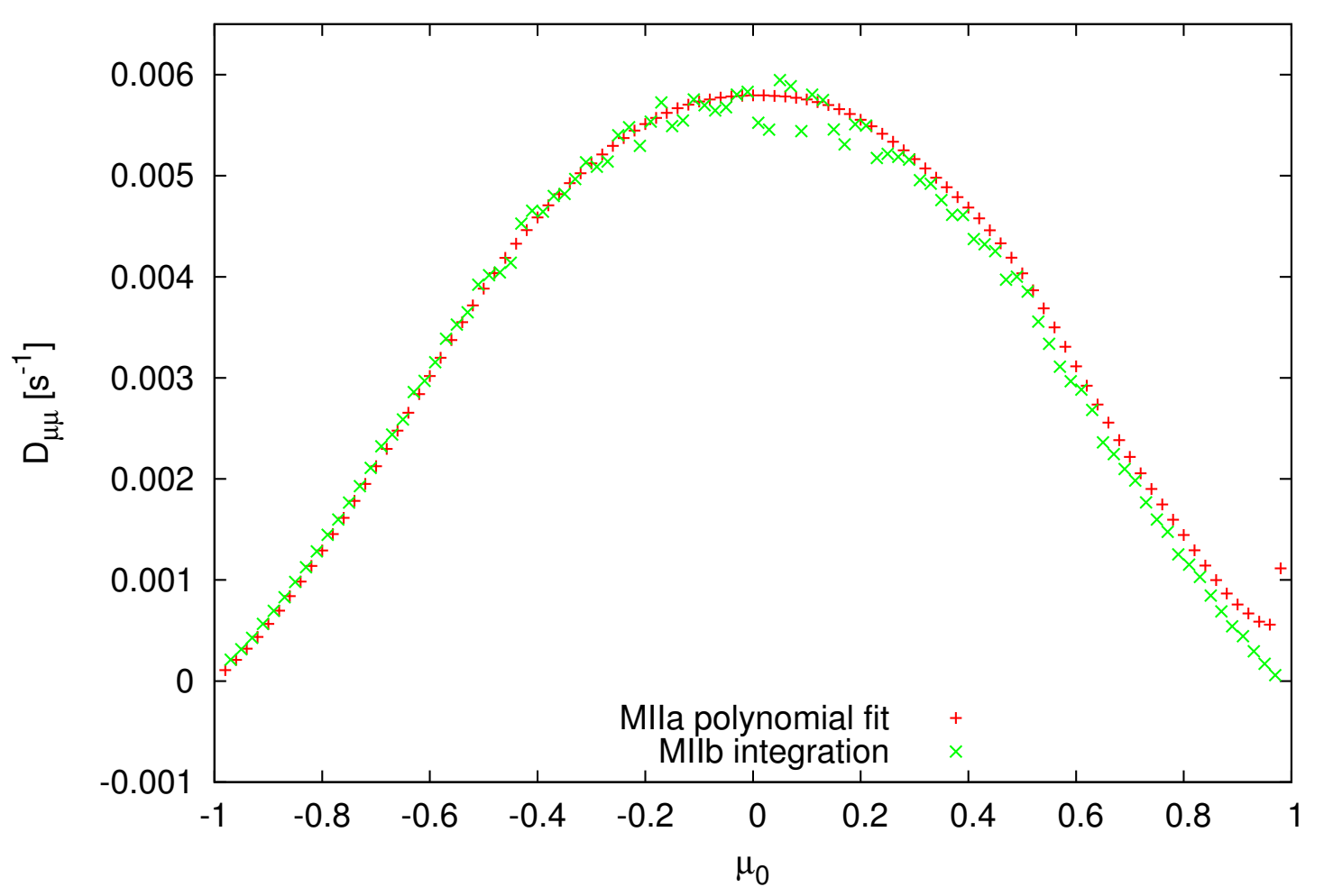

Figure 3: Pitch angle diffusion coefficient $D_{\mu \mu}$ of the Perlin-type pseudo-turbulence simulation determined with MIIa and MIIb after a runtime of 20 gyration periods.

Finally, we apply the new diffusion coefficient calculation methods MIIa nad MIIb to a Perlinnoise-type turbulence simulation in order to study its viability for large-scale particle transport as compared to MHD or PiC simulations. The Perlin noise function [9] is a method to create a randomly fluctuating variable in space with a characteristic length scale of 1 (in numerical units), and magnitudes in $[-1: 1]$. It does so without requiring any precalculated noise values, stored grids or extensive integrals, but only by interpolating pseudo-random gradient values computed on a logical coordinate grid, where only the immediate neighbours of the spatial point of interest need to be evaluated. The function can be used to create the components of the vector potential $\vec{A}(\vec{x})$ from which the magnetic field $\vec{B}(\vec{x})$ can be calculated in turn. To get a desired power-law spectrum multiple iterations of the noise can be added on top of each other.

Fig. 3 shows the resulting $D_{\mu \mu}$ that look similar to the Cherenkov resonance shapes from the MHD simulations in that it peaks around $\mu_{0}=0$ but with a significantly larger FWHM.

Again, the problem with inadequate particle statistics are visible, this time at $\mu_{0}=1$, where the mirrored half-parabola has its minimum. 


\section{Conclusions}

We have presented new methods to determine pitch angle scattering coefficients from test particle data. These methods are not limited to the QLT limit and may even be applied to observational data. Especially in the case of strong turbulence these methods, which are based on the evolution of the distribution function, may provide better results than running diffusion coefficients.

\section{Acknowledgements}

This work is based upon research supported by the National Research Foundation and Department of Science and Technology. Any opinion, findings and conclusions or recommendations expressed in this material are those of the authors and therefore the NRF and DST do not accept any liability in regard thereto.

\section{References}

[1] J. R. Jokipii, Cosmic-Ray Propagation. I. Charged Particles in a Random Magnetic Field, ApJ 146 (Nov., 1966) 480.

[2] R. Schlickeiser, Cosmic Ray Astrophysics. Springer, 2002.

[3] S. Lange and F. Spanier, Evolution of plasma turbulence excited with particle beams, A\&A $\mathbf{5 4 6}$ (Oct., 2012) A51, [arXiv:1209.0659].

[4] M. Wisniewski, F. Spanier, and R. Kissmann, Diffusion of Energetic Particles in Turbulent Magnetohydrodynamic Plasmas, ApJ 750 (May, 2012) 150, [arXiv:1203.0933].

[5] P. Kilian, T. Burkart, and F. Spanier, The influence of the mass ratio on particle acceleration by the filamentation instability, in High Performance Computing in Science and Engineering '11, pp. 5-13. Springer, Berlin Heidelberg, 2012.

[6] S. Lange, F. Spanier, M. Battarbee, R. Vainio, and T. Laitinen, Particle scattering in turbulent plasmas with amplified wave modes, A\&A $\mathbf{5 5 3}$ (May, 2013) A129, [arXiv:1303.7463].

[7] R. Vainio, T. Laitinen, and H. Fichtner, A simple analytical expression for the power spectrum of cascading Alfvén waves in the solar wind, A\&A 407 (Aug., 2003) 713-723.

[8] R. C. Tautz, A. Dosch, F. Effenberger, H. Fichtner, and A. Kopp, Pitch-angle scattering in magnetostatic turbulence. I. Test-particle simulations and the validity of analytical results, A\&A $\mathbf{5 5 8}$ (Oct., 2013) A147, [arXiv:1309.7836].

[9] K. Perlin, Improving noise, in Proceedings of the 29th Annual Conference on Computer Graphics and Interactive Techniques, SIGGRAPH '02, (New York, NY, USA), pp. 681-682, ACM, 2002. 